\title{
Matter And Gravitation In Collisions of heavy ions and neutron stars: equation of state
}

\author{
Anton Motornenko ${ }^{1,2}$, Jan Steinheimer ${ }^{1}$, Volodymyr Vovchenko ${ }^{1,2}$ \\ Stefan Schramm ${ }^{\dagger 1,2}$, and Horst Stoecker ${ }^{* 1,2,3}$ \\ ${ }^{1}$ Institut für Theoretische Physik, Goethe Universität, D-60438 Frankfurt am Main, Germany \\ ${ }^{2}$ Frankfurt Institute for Advanced Studies, Giersch Science Center, D-60438 Frankfurt am \\ Main, Germany \\ ${ }^{3}$ GSI Helmholtzzentrum für Schwerionenforschung GmbH, D-64291 Darmstadt, Germany \\ E-mail: motornenko@fias.uni-frankfurt.de
}

\begin{abstract}
The emitted gravitational waves from binary neutron star merger, as predicted from general relativistic magneto- hydrodynamics calculations, are sensitive to the appearance of quark matter and the stiffness of the equation of state of QCD Matter present in the inner cores of the stars. This is a new observable messenger from outer space, which does provide direct signals for the phase structure of strongly interacting QCD matter at high baryon density and high temperature. These astrophysically created extremes of thermodynamics do match, to within $20 \%$, the values of densities and temperatures which we find in relativistic hydrodynamics and transport theory of heavy ion collisions at the existing laboratories, if though at quite different rapidity windows, impact parameters and bombarding energies of the heavy nuclear systems. We demonstrate how one unified equation of state can be constructed and used for both neutron star physics and hot QCD matter excited at laboratory facilities. The similarity in underlying QCD physics allows the gravitational wave signals from future advanced LIGO and Virgo events be combined with the analysis of high multiplicity fluctuations and flow measurements in heavy ion detectors in the lab to pin down the EoS and the phase structure of dense matter.
\end{abstract}

Corfu Summer Institute 2018 "School and Workshops on Elementary Particle Physics and Gravity" (CORFU2018)

31 August - 28 September, 2018

Corfu, Greece
${ }^{\dagger}$ Deceased
* Speaker. 


\section{Introduction}

Modern theory of strong interactions, Quantum Chromodynamics (QCD), is challenged by two actively developing divisions of experimental physics. On one side the accelerator driven facilities are spending efforts in measuring properties of systems created in relativistic heavy ion collisions (HIC). The modern experimental laboratories, such as at the Large Hadron Collider (LHC), at the Relativistic Heavy Ion Collider (RHIC), at the Super Proton Synchrotron (SPS), and at the Heavy Ion Synchrotron (SIS), provide detailed measurements of hot strongly interacting matter that is excited in heavy ion collisions. On the other side, the phenomenology of QCD matter is accompanied by astrophysical observations of compact stars which provide insights into physics of cold but dense strongly interacting matter. The energy densities in both heavy ion collisions and in interiors of compact stars are of the same order and provide cold and hot case of dense strongly interacting matter. The recently emerged branch of observational astronomy, the gravitational wave astronomy, has established an unique interplay between hot dynamical accelerator based phenomena of heavy ion collisions and physics of cold static neutron stars. The gravitational waves emitted in general relativistic neutron star collisions, like the so far only one detected GW170817 [1], allows to study dynamics of neutron star mergers where due to dynamical compression temperatures can reach up to $100 \mathrm{MeV}[2,3]$. The QCD input into neutron star mergers is the equation of state (EoS) which provide a relation between density, temperature and pressure. The particle content and their interactions provide a basis to construct an EoS so relation to thermodynamical quantities can be obtained. Nevertheless particle content is known, the relevance of different particles in different regimes of QCD is not known and should be controlled by particle effective interactions details of which are not well established so far.

On the theoretical side there is no complete knowledge on macroscopical properties of QCD matter. It is established that at low and moderate temperatures and densities the matter can be modeled as a gas of hadrons and resonances, while it is theorized that at higher temperatures and densities the deconfinement occurs, a transition to quark and gluon degrees of freedom. Still, details of this transition are disputed, though some of them are well studied on both theoretical $[4,5,6,7]$ and experimental basis. It is still not known if the deconfinement induces a thermodynamic phase transition, this question is still open, actively discussed, and considered as main problem of QCD.

Along with the already extensively studying heavy ion collisions, neutron star mergers provide an unprecedented facility to study the dynamics of nuclear, hadronic, and possible quark matter that are excited in systems with spatial dimensions of order of $1 \mathrm{~km}$. Though matter content in both relativistic collision scenarios is very similar, there are differences due to strong gravitational effects in interiors of neutron stars and in products of their mergers.

This contribution is an attempt to show how the current knowledge of QCD phenomenology can be combined in a single framework for QCD EoS applicable for laboratory-driven and astrophysical phenomena. 


\section{An approach to QCD equation of state:}

\section{Chiral SU(3)-flavor parity-doublet Polyakov-loop quark-hadron mean-field model}

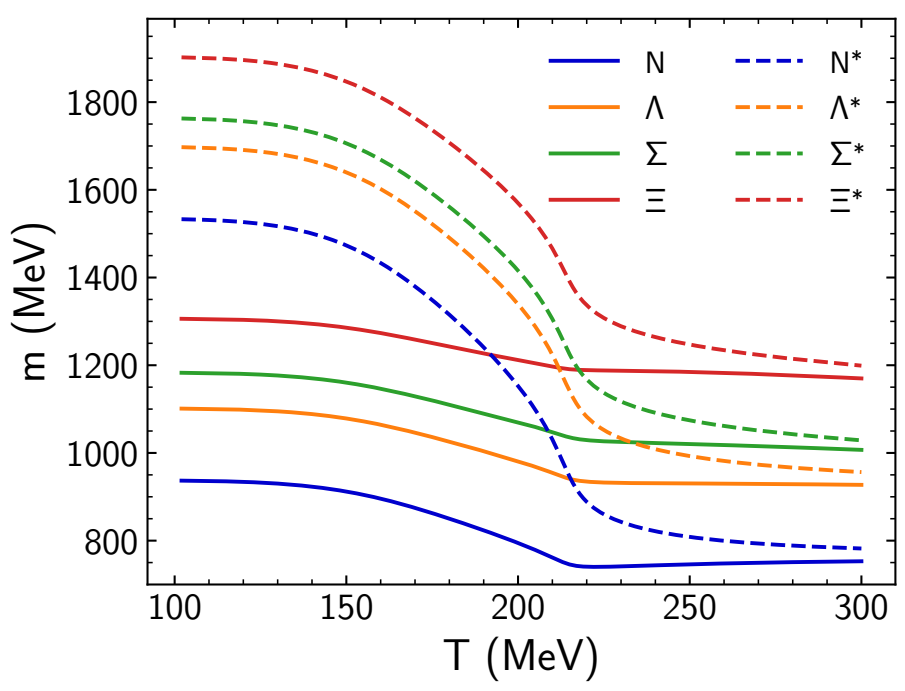

Figure 1: Effective masses of octet baryons (solid) and respective parity partners (dashed) as functions of temperature $T$ obtained from the CMF model at $\mu_{B}=0$ for isospin symmetric matter, $Q / B=\frac{1}{2}$. With the increase of the temperature the degeneracy in mass between baryons and respective parity partners arises.

The Chiral SU(3)-flavor parity-doublet Polyakov-loop quark-hadron mean-field model [8, 9], $\mathrm{CMF}$, is a modification of the $\sigma-\omega$ model to include quark degrees of freedom along with parity doubling for baryons $[10,11,12,13,14,15,8,16,17,18,19,9]$. The CMF model is an attempt to include known phenomenological QCD interactions within a unified approach to describe interacting hadron-quark matter. Essential feature of the model is the chiral symmetry restoration for baryons, the parity doubling for baryon octet states, so when chiral symmetry is restored they become degenerate in mass with respective parity partners [20,21]. Within CMF the same chiral fields generate dynamical masses for quarks, so the chiral condensate is a proxy interaction between hadrons and quarks.

The coupling parameters of baryonic sector in the model are tuned to describe empirical data on cold nuclear matter [14]. At $T=0$ the model produces nuclear ground state with the following properties: ground state density $n_{0}=0.16\left(\mathrm{fm}^{-3}\right)$, binding energy per nucleon is $E / B=-15.2$ $(\mathrm{MeV})$, asymmetry energy $S_{0}=31.9(\mathrm{MeV})$, and compressibility $K_{0}=267(\mathrm{MeV})$. The energies per baryon for nuclear matter at $T=0$ are presented in Fig. 2, where the ground state of isospin symmetric matter is presented along with the results for neutron rich matter in $\beta$-equilibrium. The CMF model can be used to provide the EoS for electric neutral neutron-rich matter in $\beta$ equilibrium, where leptons, electrons and muons, are sufficient to compensate the electric charge of hadrons, this is the matter present in interiors of neutron stars.

The hadronic sector of the CMF model is based on $\sigma-\omega$ model in mean-field description. Mesonic mean fields provide interactions between baryons, namely baryons from $\mathrm{SU}(3)_{f}$ baryon octet along with their parity partners, i.e. states with the same quantum numbers but opposite 


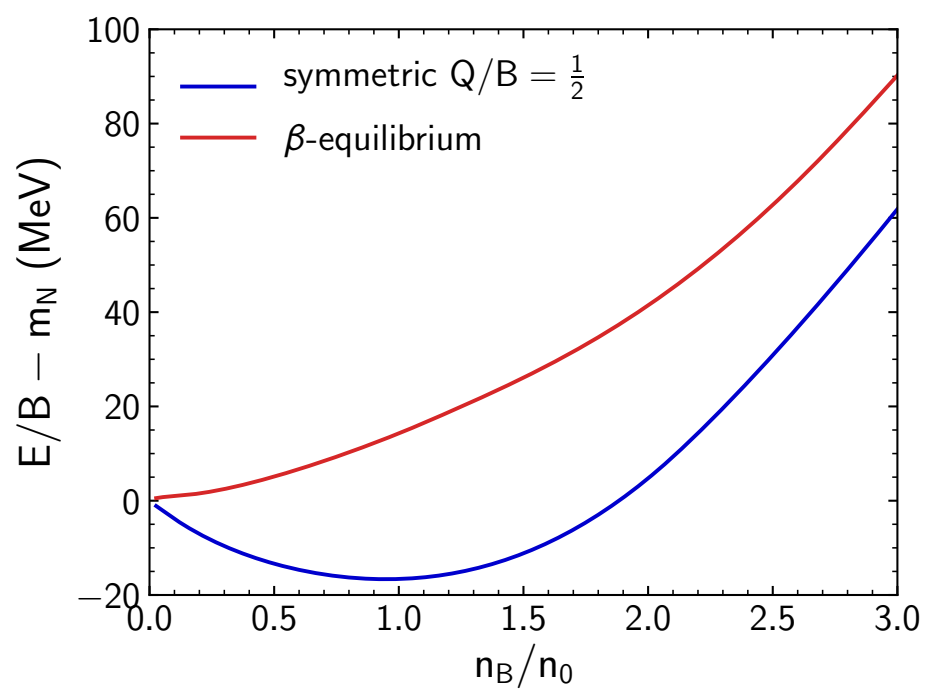

Figure 2: The CMF energy per baryon $E / B-m_{N}$ as function of baryon density $n_{B}$ normalized to nuclear saturation density $n_{0}=0.16 \mathrm{fm}^{-3}$, blue - for symmetric nuclear matter,$Q / B=\frac{1}{2}$, red - for nuclear matter in $\beta$-equilibrium, total electric charge is zero $Q=0$ that is allowed by presence of leptons.
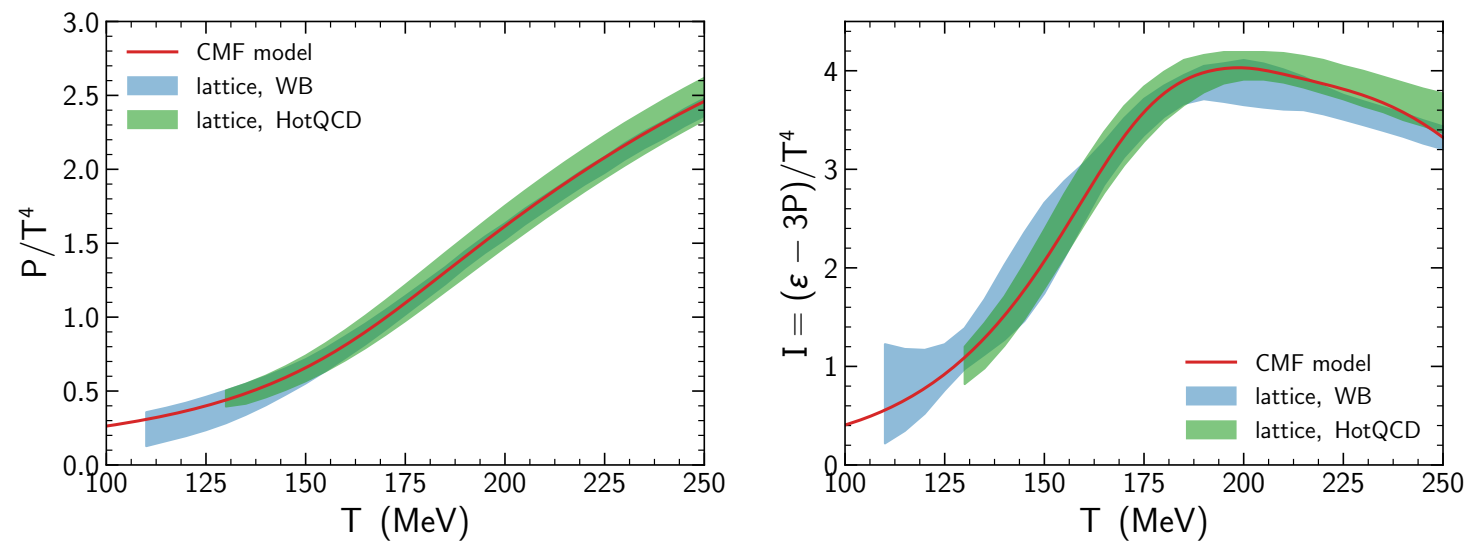

Figure 3: Pressure $P I$ (right) and trace anomaly $I$ (left) at $\mu_{B}=0$ as function of temperature $T$. Comparison between the CMF model predictions and LQCD results $[6,22]$.

parity. To provide the dynamical mass generation, the octet baryons and their partners are coupled to scalar chiral fields $\sigma$ and $\zeta$, non-strange and strange chiral condensates, respectively. $\sigma$ and $\zeta$ fields serve as order parameters for chiral transition:

$$
m_{\mathrm{i} \pm}^{*}=\sqrt{\left[\left(g_{\sigma \mathrm{i}}^{(1)} \sigma+g_{\zeta_{\mathrm{i}}}^{(1)} \zeta\right)^{2}+\left(m_{0}+n_{\mathrm{s}} m_{\mathrm{s}}\right)^{2}\right]} \pm\left(g_{\sigma \mathrm{i}}^{(2)} \sigma+g_{\zeta \mathrm{i}}^{(2)} \zeta\right) .
$$

Here + is used for positive and - is for negative parity states, $g_{\mathrm{i}}^{(\mathrm{j})}$ are the coupling constants of baryons to the two scalar fields, $m_{0}=759 \mathrm{MeV}$ is the baryon mass at the restored phase. In addition, there is an $\mathrm{SU}(3)_{f}$ symmetry-breaking mass term proportional to the strangeness content 

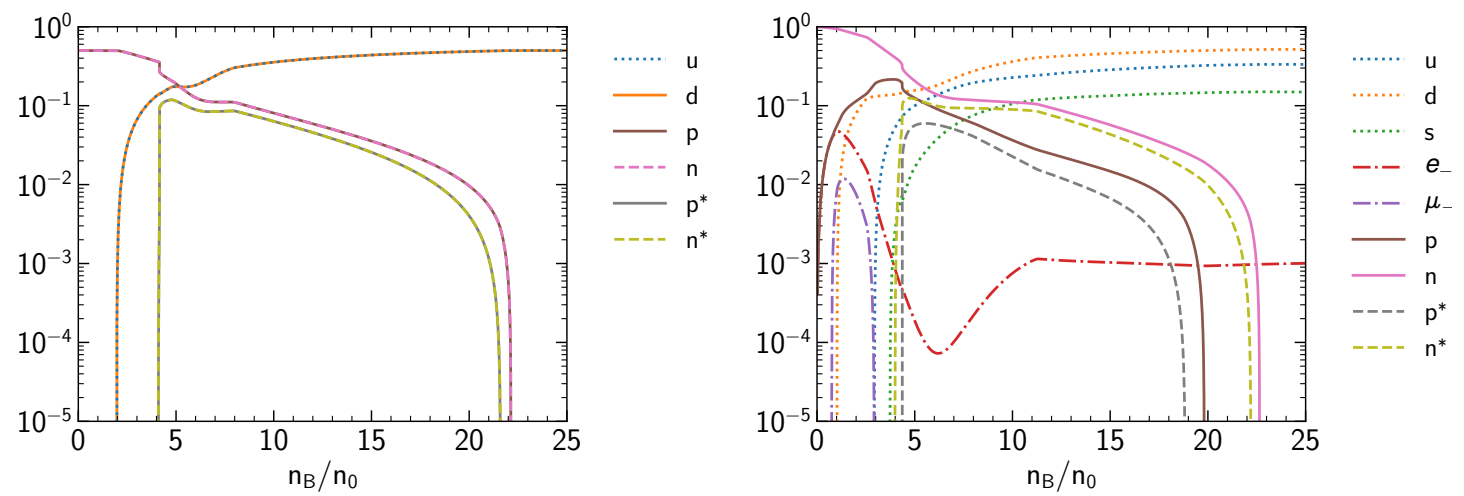

Figure 4: Particle density ratios to the density of baryons $n_{i} / n_{B}$ at $T=0$, for quarks a factor of $1 / 3$ is used, presented as functions of baryon density $n_{B}$. The CMF-results are obtained for isospin symmetric matter (left) and in $\beta$-equilibrium (right).

of the baryons, there $n_{s}$ is the number of strange quarks in the baryon, and $m_{s}=130 \mathrm{MeV}$ is the mass of the strange quark. The couplings $g_{\mathrm{i}}^{(\mathrm{j})}$ are tuned to reproduce the vacuum masses of baryons. Figure 1 presents how the mass degeneracy is restored with increasing temperature $T$ at vanishing chemical potential, $\mu_{B}=0$. At lower temperatures there is a significant mass gap between partners which diminishes as chiral symmetry restores.

The values of mesonic fields are driven by thermal potentials of quarks and by the scalar meson interaction that provides spontaneous chiral symmetry breaking:

$$
V=V_{0}+\frac{1}{2} k_{0} I_{2}-k_{1} I_{2}^{2}-k_{2} I_{4}+k_{6} I_{6}
$$

with

$$
I_{2}=\left(\sigma^{2}+\zeta^{2}\right), I_{4}=-\left(\sigma^{4} / 2+\zeta^{4}\right), I_{6}=\left(\sigma^{6}+4 \zeta^{6}\right)
$$

where $V_{0}$ is fixed by condition of vanishing potential in vacuum.

The quark sector of the model is implemented similarly with PNJL approach [23]. The thermal contribution of quarks is driven by the value of Polyakov loop $\Phi$, which plays the role of the order parameter for the deconfinement transition. Quark thermal contribution is coupled to the Polyakov loop $\Phi$ and their grand canonical potential $\Omega$ is expressed as

$$
\Omega_{q}=-T \sum_{i \in Q} \frac{d_{i}}{(2 \pi)^{3}} \int d^{3} k \ln \left(1+\Phi \exp \frac{-\left(E_{i}^{*}-\mu_{i}^{*}\right)}{T}\right)
$$

and

$$
\Omega_{\bar{q}}=-T \sum_{i \in Q} \frac{d_{i}}{(2 \pi)^{3}} \int d^{3} k \ln \left(1+\Phi^{*} \exp \frac{-\left(E_{i}^{*}+\mu_{i}^{*}\right)}{T}\right) .
$$

The sums run over all light quark flavors ( $\mathrm{u}, \mathrm{d}$, and $\mathrm{s}), d_{i}$ is the corresponding degeneracy factor, $E_{i}^{*}=\sqrt{m_{i}^{* 2}+p^{2}}$ is the energy. The quark chemical potential $\mu^{*}$ is defined by the quarks quantum numbers and is not modified by repulsive interactions as those are disfavored by lattice QCD 
(LQCD) calculations [24, 25]. Note, two- and three- quark contributions to $\Omega$ are omitted in the CMF model since hadronic excitations are explicitly included.

The effective masses of the light quarks are generated by the $\sigma$ and $\zeta$, non-strange and strange chiral condensates respectively. The quark explicit mass terms $\delta m_{q}=5 \mathrm{MeV}$, and $\delta m_{s}=150 \mathrm{MeV}$ for the strange quark, and $m_{0 q}=253 \mathrm{MeV}$ correspond to an explicit mass term which does not originate from chiral symmetry breaking:

$$
\begin{aligned}
& m_{q}^{*}=-g_{q \sigma} \sigma+\delta m_{q}+m_{0 q}, \\
& m_{s}^{*}=-g_{s \zeta} \zeta+\delta m_{s}+m_{0 q} .
\end{aligned}
$$

Dynamics of the Polyakov-loop is controlled by the effective Polyakov-loop potential $U\left(\Phi, \Phi^{*}, T\right)[26]$ :

$$
\begin{gathered}
U=-\frac{1}{2} a(T) \Phi \Phi^{*}+b(T) \log \left[1-6 \Phi \Phi^{*}+4\left(\Phi^{3}+\Phi^{* 3}\right)-3\left(\Phi \Phi^{*}\right)^{2}\right] \\
a(T)=a_{0} T^{4}+a_{1} T_{0} T^{3}+a_{2} T_{0}^{2} T^{2}, b(T)=b_{3} T_{0}^{4}
\end{gathered}
$$

The parameters of this potential are fixed to the lattice QCD data on the interaction measure $I=\frac{\varepsilon-3 P}{T^{4}}$ [9]. Figure 3 shows a comparison between the CMF predictions for $\mu_{B}=0$ thermodynamics and LQCD data $[6,22]$. The parameter values of $U\left(\Phi, \Phi^{*}, T\right)$ ensure applicability of the CMF model at high temperatures, so fair description of LQCD data is possible within one unified approach for both hot QCD thermodynamics and cold nuclear matter description.

Figure 4 demonstrates particle content at $T=0$ for isospin symmetric matter and for matter in $\beta$-equilibrium. At $n_{B} \approx 4 n_{0}$ the phase transition associated with chiral symmetry restoration takes place. At that region parity partners become degenerate in mass so degeneracy factors of nucleons are effectively increased twice. Note, that at $T=0$ baryons are presented only by nucleons and their parity partners. Though heavier baryons and their resonances are present in the model, at $T=0$ they are suppressed by strong repulsion and they start to appear only with the increase of temperature. After chiral symmetry restoration a significant fraction of quarks is present, so within the CMF model there's no phase transition that separates quark and hadron phases. Only at very high densities $n_{B} \gtrsim 22 n_{0}$ the content of the matter is composed from only quarks. This thermodynamic transition is of the second order.

To mimic hard-core repulsive interactions among baryons, the excluded-volume effects are incorporated within the CMF model [16]. This approach ensures that quarks and gluons dominate at high densities and temperatures. This results that at all particle densities, including quarks, are reduced as parts of the system are occupied by EV-hadrons:

$$
\rho_{j}=\frac{\rho_{j}^{\mathrm{id}}\left(T, \mu_{j}^{*}-v_{j} p\right)}{1+\sum_{i}^{\mathrm{HRG}} v_{i} \rho_{i}^{\mathrm{id}}\left(T, \mu_{i}^{*}-v_{i} p\right)},
$$

the $v_{i}$ are the eigenvolume coefficients for different species. $p$ is pressure of the system without contribution of mean fields, $\mu^{*}$ denotes modified chemical potential of the hadron. The $v$ is fixed to $v_{B}=1 \mathrm{fm}^{3}$ for (anti-) baryons, $v_{M}=1 / 8 \mathrm{fm}^{3}$ for mesons, and is set to zero $v_{q}=0$ for quarks, indices $i$ and $j$ run through all states in hadron-resonance gas and, additionally, quarks. 


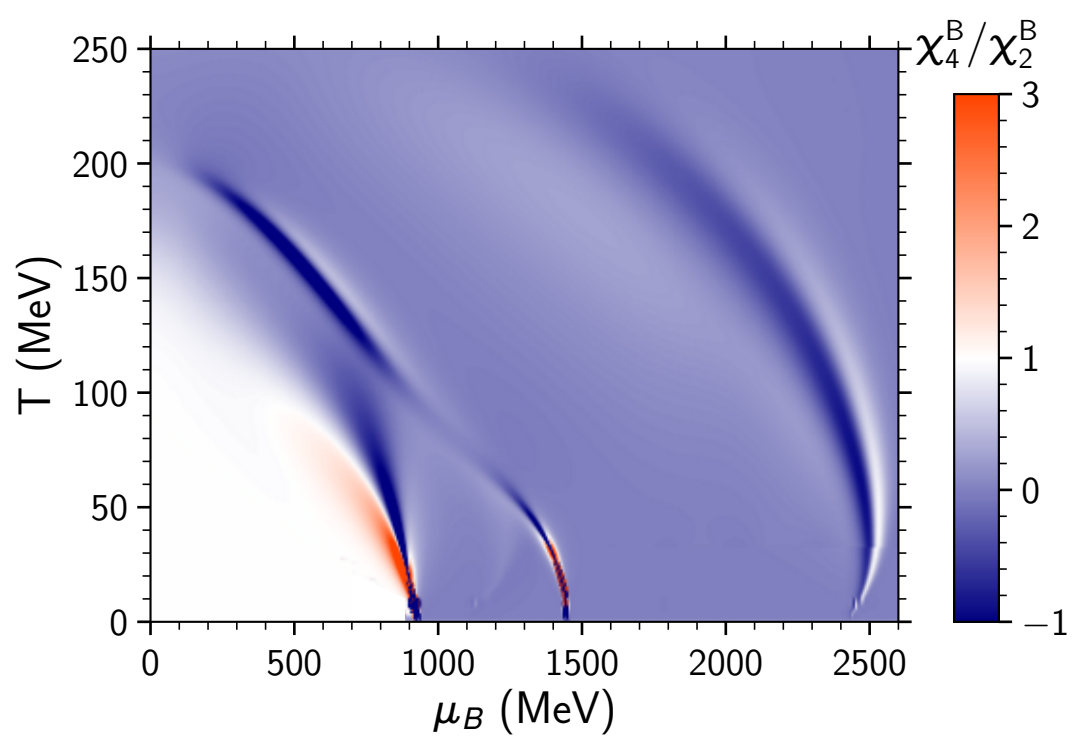

Figure 5: $\mathrm{CMF}$ baryon number kurtosis, ratio of fourth and second order susceptibilities, $\chi_{4}^{\mathrm{B}} / \chi_{2}^{\mathrm{B}}$ in the baryon chemical potential and temperature, $\mu_{\mathrm{B}}-T$, plane. The three distinctive critical regions and their remnants spread from $T=0$ up to $T>200 \mathrm{MeV}$.

\section{The CMF model's QCD phase diagram}

Numerous phenomenological features of the CMF model suggest a rather rich phase structure of the model. The critical phenomena associated with the nuclear liquid-vapor phase transition, chiral symmetry restoration and quark appearance that are present in the CMF model provide a complicated interplay. To determine location of different phases the baryon number susceptibilities $\chi_{n}^{B}$ are used:

$$
\chi_{n}^{B}=\frac{\partial^{n}\left(P / T^{4}\right)}{\left(\partial \mu_{B} / T\right)^{n}}
$$

Higher-order baryon number susceptibilities are measures of baryon number fluctuations in grand canonical ensemble. $\chi_{n}^{B}$ rise proportionally to the increasing powers of the correlation length [27], so increase in correlation length is reflected in large values of 2 nd and higher-order susceptibilities in the vicinity of critical point and in the region of phase transition or crossover. Hence, these quantities are useful indicators of a critical behavior. Deviations of $\chi_{n}^{B}$ from the corresponding baselines indicate a transformation between different phases, which is reflected usually in a nonmonotonic behavior of these observables, e.g. kurtosis $\chi_{4}^{B} / \chi_{2}^{B}$.

The CMF model kurtosis, $\chi_{4}^{B} / \chi_{2}^{B}$, Fig. 5, indicates several structures in $\mu_{B}-T$ plane. Those structures are attributed to thermal behavior of various order parameters that experience rapid change in these regions.

At low and intermediate temperatures and chemical potentials rapid growth in baryonic density provides a change in value of kurtosis' baseline from 1 to 0 . This transition is associated with nuclear liquid-vapor phase transition, that, at low temperatures, is a phase transition from dilute gas of nucleons to dense nuclear matter. This transition smoothens out to crossover at $T \gtrsim 20$ 


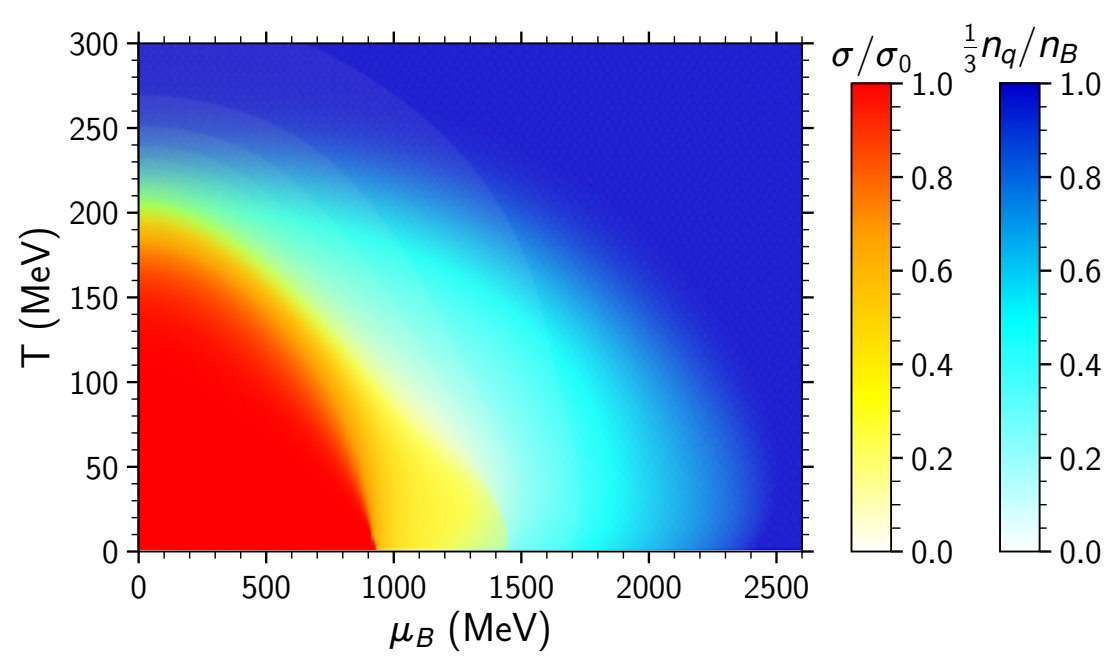

Figure 6: Colormaps for chiral condensate $\sigma$ and quark fraction $\frac{1}{3} n_{q} / n_{B}$ along the $\mu_{B}-T$ plane for isospin symmetric matter. Note that significant quark fraction appears only after chiral symmetry is restored, so pure quark matter arises at high $\mu_{B}$ or $T$. Mind the transparency of white color, so two quantities are presented on the same plot.

$\mathrm{MeV}$, so change in baryon density is smooth and not step-like. Note, even at $\mu_{B}=0$ and $T>100$ $\mathrm{MeV}$ the remnants of nuclear liquid-vapor transition are significant and still contribute to critical behavior of the CMF model.

At higher temperatures and chemical potentials the second consecutive critical region is attributed to chiral symmetry restoration. This transition is driven by rapid vanishing of chiral condensate $\sigma$, see Fig. 6 , so degeneracy among parity partners is provided and quark masses are decreased. This transition is of a first order with critical temperature $T_{\mathrm{CP}}^{\text {chiral }} \approx 20 \mathrm{MeV}$, so chiral condensate drops discontinuously at $T<T_{\mathrm{CP}}^{\text {chiral }}$, at higher temperatures this transition is crossovertype. Only after the chiral symmetry restoration the quark fraction has significant values in the CMF model. The free quark contribution to baryon density, $\frac{1}{3} n_{q} / n_{B}$, is presented in Fig. 6 .

Quarks in the model appear smoothly, so there is no deconfinement related phase transition. Quarks appear in chirally restored phase and they are in mixture with other baryons and mesons. This smooth appearance of free quarks and their presence along with other baryons is similar to Quarkyonic matter model [5, 28] where quarks appear from Fermi sea and Baryons reside on the Fermi shell.

Only at sufficiently high temperatures or large baryon densities the content of the matter is only contributed by quarks and gluons within Polyakov loop potential. This transition produces third consecutive critical region in kurtosis along $\mu_{B}-T$ plane. This transition is of a second order and is driven by the reduction of degrees of freedom, hadrons are no longer contributing to the thermodynamics.

\section{Probing the phase diagram by heavy ion collisions}

During its expansion the system exited in heavy ion collision probes various regions of the phase diagram. The trajectory of the system evolution can be estimated by hydrodynamic mod- 
eling. The input to hydro simulations is the initial stage that is produced in rapid violent nuclear collision. In the initial state the entropy is produced by the violent shock compression [29]. While system cools down during expansion, the entropy increases only moderately due to the rather small viscosity [30, 31], thus an isentropic expansion scenario is a reasonable approximation [32].

We illustrate which regions of the phase diagram are probed in heavy ion collisions at low and moderate energies by using one dimensional Taub adiabat model [33, 34, 35]. This model describes the expansion by lines of constant entropy per baryon $S / B=$ const (isentropes). These lines describe the isentropic matter evolution of ideal fluid dynamics at different collision energies.

The isentropic expansion of the equilibrated matter continues until the system becomes so dilute that the chemical as well as the kinetic freeze-out occur when the chemical composition is fixed.

The calculation of initial entropy per baryon $(S / A)$ is done in the assumption of 1-dimensional stationary scenario of central HIC - the two colliding slabs of cold nuclear matter [36, 34, 37, 38, 39, 40, 29]. The relativistic Rankine-Hugoniot equation (Taub adiabat), RRHT, [33, 35] provide conservation of the baryon number, energy and momentum across the shock front. Using RRHT entropy is directly associated to the collision energy. The RRHT-equation provide thermodynamic properties across the shock front

$$
\left(P_{0}+\varepsilon_{0}\right)\left(P+\varepsilon_{0}\right) n^{2}=\left(P_{0}+\varepsilon\right)(P+\varepsilon) n_{0}^{2},
$$

where $P_{0}, \varepsilon_{0}$ and $n_{0}$ correspond to the initial pressure, energy density, and baryon density in the local rest frame of each of the two slabs. The two symmetric slabs consist of the nuclear matter in the ground state, $P_{0}=0, \varepsilon_{0} / n_{0}-m_{N}=-16 \mathrm{MeV}$ and $n_{0}=0.16 \mathrm{fm}^{-3}$. The created density is related to collision energy as:

$$
\gamma^{\mathrm{CM}}=\frac{\varepsilon n_{0}}{\varepsilon_{0} n}, \gamma^{\mathrm{CM}}=\sqrt{\frac{1}{2}\left(1+\frac{E_{\mathrm{lab}}}{m_{N}}\right)} .
$$

Here $\gamma^{\mathrm{CM}}$ is the Lorentz gamma factor in the center of mass frame of the HIC and $E_{\mathrm{lab}}$ is the beam energy per nucleon in the laboratory frame of a fixed target collision. This relation can be obtained from the full stopping condition [34, 37, 38, 39, 40, 29, 41]. The initial state thermodynamics (density, temperature and entropy) of the hot, dense participant matter is obtained from Eqs. (4.1) and (4.2) as a function of the collision energy. The known initial entropy yields the lines of constant entropy which give the trajectories of the heavy ion collisions in the phase diagram.

The predicted isentropic expansion trajectories are shown in the $\mu_{B}-T$ phase diagram in Fig. 7.

The RRHT-adiabat scenario predicts a very strong compression and heating already at intermediate lab (fixed target) bombarding energies. The hot and dense system passes the chiral transition predicted by the present CMF model already at $E_{\text {lab }} \approx 2 \mathrm{~A} \mathrm{GeV}$, i.e. at energies available at GSI's SIS18 accelerator facility. Here the specific total entropy is predicted to reach $S / A \approx 3$, in accord with previous RMF-calculations [40] which also used the 1-D RRHT-scenario. The $\mu_{B}-T$ values, $T \approx 70 \mathrm{MeV}, \mu_{B} \approx 1.2 \mathrm{GeV}$, with net baryon densities $n_{B} / n_{0} \approx 3$, reached here in HIC, coincide with the $\mu_{B}-T$ values reached in binary neutron star collisions, as recent general relativistic fully 3+1-dimensional megneto-hydrodynamical calculations have confirmed [42, 2] for the gravitational wave event GW170817. At these temperatures and densities, $T \approx 70 \mathrm{MeV}$ and $n_{B} / n_{0} \approx 3$, 

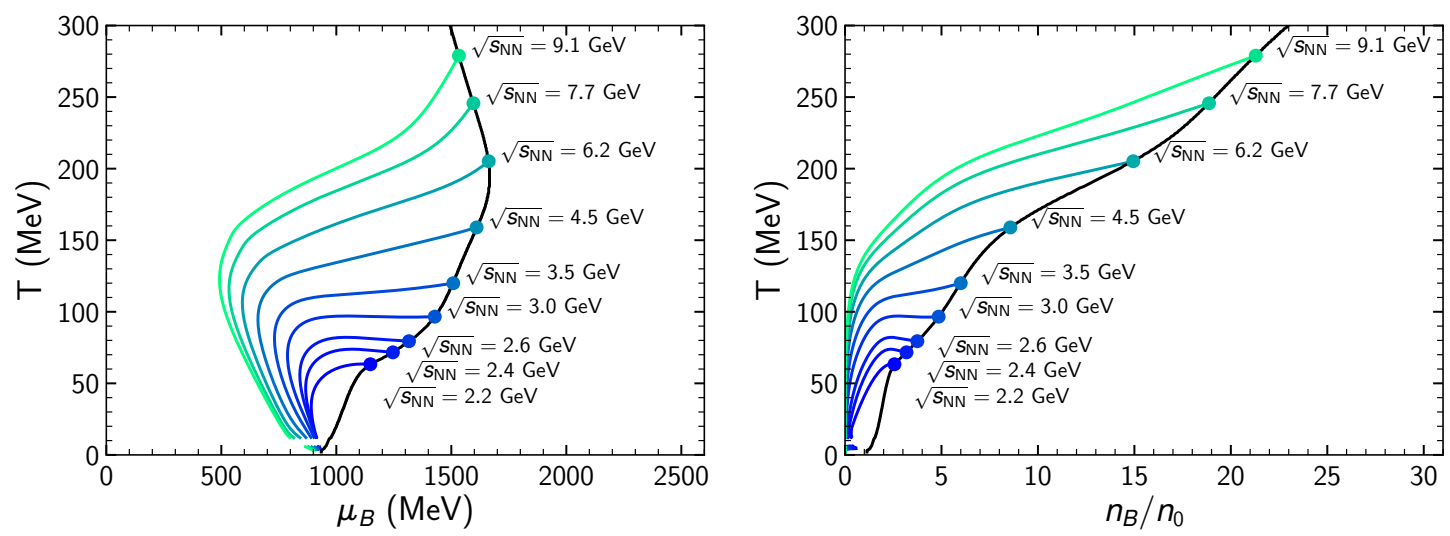

Figure 7: Evolution for different collision energy $\sqrt{s_{\mathrm{NN}}}$ of system excited in heavy-ion collisions along the $\mu_{B}-T$ (left) and $T-n_{B}$ (right) phase diagram. Initial state as implicit function of $\sqrt{s_{\mathrm{NN}}}$ is calculated by Taub adiabat and is presented by the black line. Colored lines - isentropic lines of constant entropy per baryon $S / A$ at different bombarding energies $\sqrt{s_{N N}}$ respectively.

the RRHT model predicts that there are about $20 \%$ of the dense matter are already transformed to quarks.

Heavy ion fixed target experiments of SIS at FAiR and SPS at CERN as well as STAR BES program at RHIC probe temperatures from $50<T<280 \mathrm{MeV}$ and chemical potentials from $500<$ $\mu_{B}<1700 \mathrm{MeV}$ for the collision energy range $\sqrt{s_{\mathrm{NN}}}<10 \mathrm{GeV}$ considered here. In this region the CMF model shows not an additional phase transition, but the remnants of the nuclear liquidvapor transition at $T \approx 20 \mathrm{MeV}$. The chiral transition at larger chemical potentials can influence the dynamical evolution, too. The present results suggest that heavy-ion collisions mostly probe regions where the nuclear matter liquid-vapor critical point dominates - hence, the observed baryon fluctuations are largely due to remnants of the nuclear liquid-vapor phase transition. This had been suggested also in previous works $[43,18,44,45]$. The CP associated with the chiral symmetry restoration in the CMF model lies at $\mu_{B} \approx 1.5 \mathrm{GeV}$ and $T \approx 20 \mathrm{MeV}$. This high density region is to the best of our knowledge is reachable in the interior of neutron stars, NS, and in binary general relativistic NS mergers [46, 47, 2, 3, 48, 49].

\section{QCD equation of state and neutron stars}

Interiors of neutron stars consist of matter that several times surpass nuclear saturation density. Theory of matter of at these densities is not complete and details of the equation of state are missing. As discussed above, the situation is similar to the matter created in HIC where details of equation of state at high temperatures are not known. There are ongoing discussions of relevance of hyperonic, quark, and hadronic degrees of freedom in cold and dense neutron star matter.

The CMF model is a general purpose equation of state that with the same set of parameters can be applied to describe the neutron star matter. To produce the equation of state for compact stellar objects the condition of electric neutrality is imposed so $\beta$-equilibrium is ensured, these constraints demand the presence of leptons. This changes the particle content as depicted at Fig. 4. As a result, 

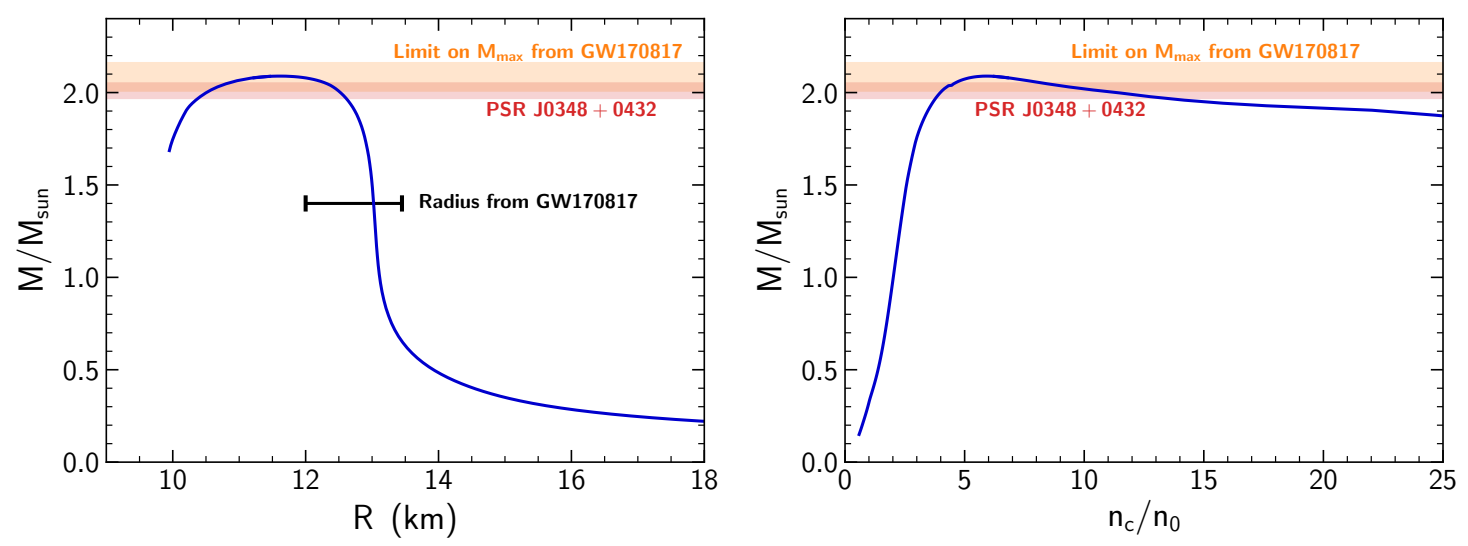

Figure 8: Mass-radius relation (left) and mass - central density relation (right) for neutron stars calculated using the CMF equation of state. Yellow colorbands present constraint on maximum mass of neutron star obtained in [53], red colorband - mass of neutron star PSR J0348+0432 [54].

strangeness and hypercharges obtain finite non-zero values and quark-hadron degrees of freedom are present as well. The temperatures in the star interiors are negligibly small compared to hot QCD scales, so $T=0$ EoS can be applied.

The resulting CMF EoS at $T=0$ is used as the input for the Tolman-Oppenheimer-Volkoff (TOV) equation, so relation between the mass and the radius can be obtained for any static, spherical, gravitationally bound object $[50,51]$, i.e. here a static neutron star, NS. The content of neutron stars' surface layers presumably consist of mostly neutron rich nuclei and clusters in chemical and $\beta$ - equilibrium. Those nuclei are not yet a part of the CMF model. Therefore, we use the classical crust-EoS [52] matched additionally to the CMF-EoS at $n_{B} \approx 0.05 \mathrm{fm}^{-3}$.

The NS mass-radius relation obtained using TOV equation with the CMF EoS is presented in Fig. 8. The discussion of quark content of the stars is presented in [9]. Note that unstable branch of mass radius diagram is created by stars where quark contribution to baryon density is $30 \%$ and more. The central densities of the stable stars can not exceed $n_{B}=6 n_{0}$, as shown in the right panel of Fig. 8. On the Fig. 8 the maximum mass indicates the "last stable star". The continuous monotonous transition from NS matter to a deconfined quark phase provides a smooth appearance of quarks in the star structure and prevents a "second family" of stable solutions. Therefore there is no strict separation between a quark core and the hadronic interior of the star. This is a CMF result due to the Polyakov loop implementation of the deconfinement mechanism and no vector repulsion among quarks. Though LQCD data disfavors repulsive forces for quarks, there is active discussion concerning vector repulsion in dense baryonic matter in neutron star interiors $[55,56]$.

During the binary neutron star mergers the colliding participants experience significant nonspherical gravitational fields induced by their merge companions. These modifications of gravitational field induce tidal deformations of the stars. The response of a neutron star to the nonspherical field depends strongly on EoS and is reflected in the tidal deformability coefficient $\lambda$ [57]. The tidal deformability $\lambda$ is a measure of the induced quadruple moment $Q_{i j}$ in a response to the external tidal field $\mathscr{E}_{i j}$ :

$$
Q=-\lambda \mathscr{E}_{i j}
$$



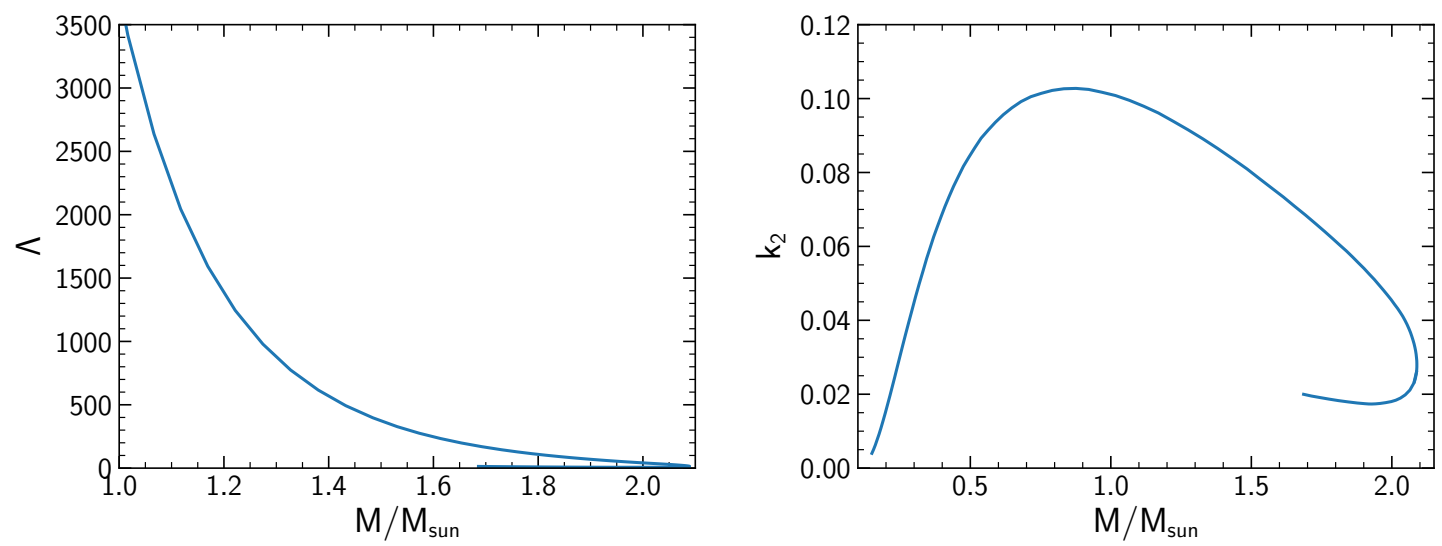

Figure 9: The CMF tidal deformability $\Lambda$ (left) and second Love number $k_{2}$ (right) as functions of NS mass.

$\lambda$ is directly proportional to the second Love number $k_{2}$ :

$$
\lambda=\frac{2}{3} k_{2} R^{5} .
$$

For convenience, usually the dimensionless tidal deformability $\Lambda$ is presented as:

$$
\Lambda=\frac{\lambda}{M^{5}}=\frac{2}{3} k_{2}\left(\frac{R}{M}\right)^{5} .
$$

Here, $M$ and $R$ are the mass and radius of the neutron star. We present resulting dimensionless tidal deformability coefficient $\Lambda$ and second Love number $k_{2}$ in Fig. 9, see Ref. [9] for discussion.

Fig. 10 shows the evolution of the baryon density $n_{B}$ and temperature $T$ in heavy ion collisions and regions accessible in neutron star mergers. The evolution of HIC is calculated in detailed 3D hydrodynamic simulation as well as in simplified 1D calculation with RRHT adiabat. The regions reachable in NS mergers are obtained using general relativistic magneto-hydrodynamic simulations [58, 48, 49]. The figure illustrates similarities between hot and dense matter created in NS mergers and heavy ion collisions. The collisions have similarities due to rapid collision and compression of nuclear matter and differences due to spatial structure of neutron stars and presence of gravitational forces.

During the merger the neutron stars touch each other by their outer regions where densities are smaller than saturation density. At initial stage of the merger these regions are rapidly compressed and heated, the hot NS regions appear shortly after $t \approx 1 \mathrm{~ms}$. These regions are indicated by red in Fig. 10, temperatures in these merged outer layers reach up to $T \approx 75 \mathrm{MeV}$. At the early stage of the merger there are two distinct dense regions that correspond to NS cores. After the initial heating the differences between NS collisions and HIC emerge. The blue region in Fig. 10 is compressed much slower and is not heated so much. As the merger evolves further the two dense cold cores slowly move towards each other as a result of gravitation attraction. At the time around $t \approx 5 \mathrm{~ms}$ these two cold cores approach each other and form a dense and slightly heated, $T \lesssim 20 \mathrm{MeV}$, core of newly formed rotating hypermassive neutron star. The hot region created at initial stage is split in two by the two approaching each other cores, so at $t \approx 5 \mathrm{~ms}$ there are two hot spots and one 


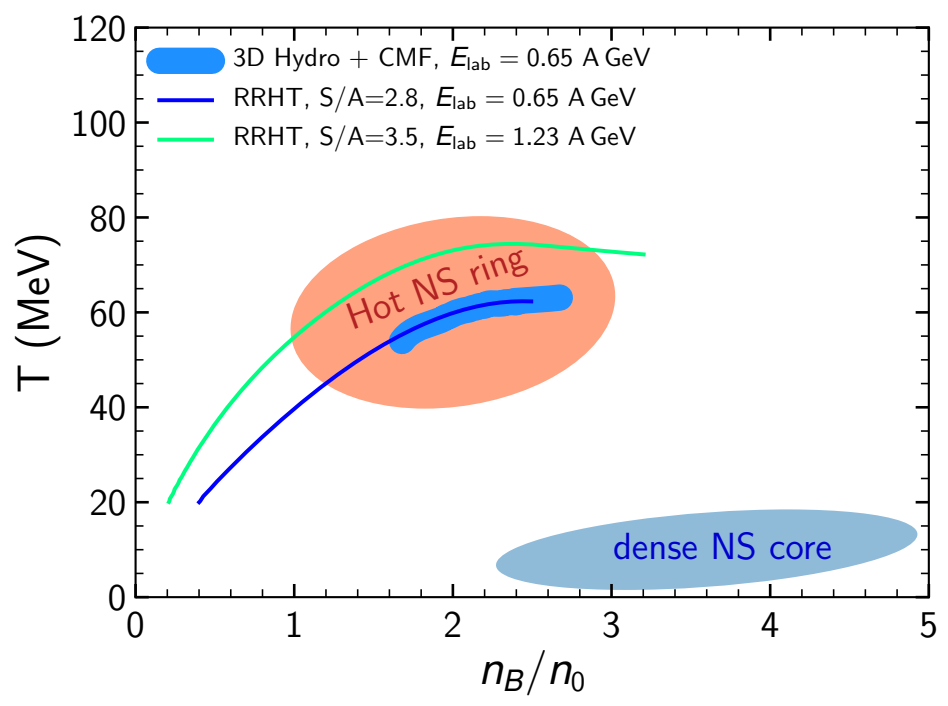

Figure 10: Regions of $n_{B}-T$ phase diagram reachable in lower energy heavy ion collisions and in neutron star mergers. Bold line present results from full 3D hydrodynamic simulation with CMF EoS used. Thin lines indicate isentropes where entropy per baryon, $S / A$ was calculated by RRHT adiabat. Red and blue ellipses indicate regions of maximal temperature and density respectively, reachable in neutron star mergers, extracted from [48].

dense cold core. During the further evolution these two hot spots rotate around the central core and at $t \gtrsim 15 \mathrm{~ms}$ smear out into hot NS ring. So at late postmerger stages the central region of hypermassive rotating NS is cold and dense and the middle interiors of NS consist from hot matter, blue and red regions of Fig. 10 respectively [58, 48, 49].

\section{Summary}

The QCD equation of state consistent with LQCD, heavy ion and neutron star physics is presented. The Chiral SU(3)-flavor parity-doublet Polyakov-loop quark-hadron mean-field model, $\mathrm{CMF}$, is a phenomenological approach to QCD thermodynamics with quark and hadron degrees of freedom. The QCD phase diagram calculated in CMF includes three critical regions: nuclear liquid vapor phase transition, phase transition relation related to chiral symmetry restoration, and second order transition to pure quark matter. In CMF quarks appear smoothly without discontinuities in quark fraction. After chiral symmetry restoration takes place a significant quark fraction is present within the model, at chirally restored phase there is a mixture of quarks and hadrons. With the increase of temperature or density in chirally restored phase, quark fraction monotonously rises until sudden appearance of quark matter. There baryonic density is contributed only by free quarks, this transition is always of second order.

The variety of QCD phenomena accommodated in the CMF model allows to reproduce LQCD data, neutron star astrophysical observables and apply the same EoS to heavy ion physics. This illustrates the similarity of underlying QCD physics in these physical phenomena and suggests that QCD matter can be probed experimentally not only in laboratory-based accelerator facilities, 
but also in stable neutron star observations and dynamical mergers of neutron stars. The later are observable by gravitational wave signals detectable at LIGO and VIRGO laboratories.

\section{Acknowledgements}

We acknowledge fruitful discussions with Matthias Hanauske, Elias Most, Jens Papenfort, and Luciano Rezzolla. The authors thank HIC for FAIR, HGS-HIRe for FAIR, BMBF, and DFG for support. J.S. appreciates the support of the SAMSON AG, WGG-Forderverein, and the C.W. Fück-Stiftungs Prize 2018. H.St. acknowledges the support through the Judah M. Eisenberg Laureatus Chair at Goethe University, and the Walter Greiner Gesellschaft, Frankfurt. Computational resources have been provided by the Center for Scientific Computing (CSC) at the J. W. GoetheUniversity, Frankfurt.

\section{References}

[1] LigO Scientific, Virgo collaboration. Phys. Rev. Lett. 116 (2016) 061102 [1602.03837].

[2] E. R. Most, L. J. Papenfort, V. Dexheimer, M. Hanauske, S. Schramm, H. Stoecker et al. Phys. Rev. Lett. 122 (2019) 061101 [1807.03684].

[3] A. Bauswein, N.-U. F. Bastian, D. B. Blaschke, K. Chatziioannou, J. A. Clark, T. Fischer et al. Phys. Rev. Lett. 122 (2019) 061102 [1809.01116].

[4] E. V. Shuryak. Phys. Rept. 61 (1980) 71.

[5] L. McLerran and R. D. Pisarski. Nucl. Phys. A796 (2007) 83 [0 706 . 2191].

[6] S. Borsanyi, Z. Fodor, C. Hoelbling, S. D. Katz, S. Krieg and K. K. Szabo. Phys. Lett. B730 (2014) $99[1309.5258]$.

[7] HotQCD collaboration. Phys. Rev. D96 (2017) 074510 [1708. 04897 ].

[8] J. Steinheimer, S. Schramm and H. Stoecker. J. Phys. G38 (2011) 035001 [1 009.5239 ].

[9] A. Motornenko, J. Steinheimer, V. Vovchenko, S. Schramm and H. Stoecker. . 1905.00866.

[10] C. E. Detar and T. Kunihiro. Phys. Rev. D39 (1989) 2805.

[11] T. Hatsuda and M. Prakash. Phys. Lett. B224 (1989) 11.

[12] P. Papazoglou, J. Schaffner, S. Schramm, D. Zschiesche, H. Stoecker and W. Greiner. Phys. Rev. C55 (1997) 1499 [nucl-th/9609035].

[13] P. Papazoglou, S. Schramm, J. Schaffner-Bielich, H. Stoecker and W. Greiner. Phys. Rev. C57 (1998) $2576[$ nucl-th/9706024].

[14] P. Papazoglou, D. Zschiesche, S. Schramm, J. Schaffner-Bielich, H. Stoecker and W. Greiner. Phys. Rev. C59 (1999) 411 [nucl-th/9806087].

[15] C. Sasaki and I. Mishustin. Phys. Rev. C82 (2010) 035204 [1 005 . 4811].

[16] J. Steinheimer, S. Schramm and H. Stoecker. Phys. Rev. C84 (2011) 045208 [11 08 . 2596 ].

[17] V. Dexheimer, J. Steinheimer, R. Negreiros and S. Schramm. Phys. Rev. C87 (2013) 015804 [1206.3086]. 
[18] A. Mukherjee, J. Steinheimer and S. Schramm. Phys. Rev. C96 (2017) 025205 [1611.10144].

[19] A. Motornenko, V. Vovchenko, J. Steinheimer, S. Schramm and H. Stoecker. Nucl. Phys. A982 (2019) 891 [1809.02000].

[20] G. Aarts, C. Allton, D. De Boni, S. Hands, B. Jäger, C. Praki et al. JHEP 06 (2017) 034 [1703.09246].

[21] G. Aarts, C. Allton, D. De Boni and B. Jäger. . 1812.07393.

[22] HotQCD collaboration. Phys. Rev. D90 (2014) 094503 [1407.6387].

[23] K. Fukushima. Phys. Lett. B591 (2004) 277 [hep-ph/0310121].

[24] J. Steinheimer and S. Schramm. Phys. Lett. B696 (2011) 257 [1 005 . 1176].

[25] J. Steinheimer and S. Schramm. Phys. Lett. B736 (2014) 241 [1401. 4051 ].

[26] C. Ratti, M. A. Thaler and W. Weise. Phys. Rev. D73 (2006) 014019 [hep-ph/ 0506234 ].

[27] M. A. Stephanov. Phys. Rev. Lett. 102 (2009) 032301 [0809. 3450 ].

[28] L. McLerran and S. Reddy. Phys. Rev. Lett. 122 (2019) 122701 [1811.12503].

[29] H. Stoecker and W. Greiner. Phys. Rept. 137 (1986) 277.

[30] L. P. Csernai, J. Kapusta and L. D. McLerran. Phys. Rev. Lett. 97 (2006) 152303 [nucl-th/0604032].

[31] P. Romatschke and U. Romatschke. Phys. Rev. Lett. 99 (2007) 172301 [0 706.1522 ].

[32] J. Steinheimer, M. Bleicher, H. Petersen, S. Schramm, H. Stoecker and D. Zschiesche. Phys. Rev. C77 (2008) 034901 [0710.0332].

[33] A. H. Taub. Phys. Rev. 74 (1948) 328.

[34] H. Stoecker, W. Greiner and W. Scheid. Z. Phys. A286 (1978) 121.

[35] K. S. Thorne. 179 (1973) 897.

[36] H. G. Baumgardt, J. U. Schott, Y. Sakamoto, E. Schopper, H. Stoecker, J. Hofmann et al. Z. Phys. A273 (1975) 359.

[37] H. Stoecker, G. Graebner, J. A. Maruhn and W. Greiner. Phys. Lett. 95B (1980) 192.

[38] H. Stoecker, A. A. Ogloblin and W. Greiner. Z. Phys. A303 (1981) 259.

[39] H. Stoecker, M. Gyulassy and J. Boguta. Phys. Lett. 103B (1981) 269.

[40] D. Hahn and H. Stoecker. Nucl. Phys. A476 (1988) 718.

[41] A. V. Merdeev, L. M. Satarov and I. N. Mishustin. Phys. Rev. C84 (2011) 014907 [11 03.3988 ].

[42] M. Hanauske, J. Steinheimer, L. Bovard, A. Mukherjee, S. Schramm, K. Takami et al. J. Phys. Conf. Ser. 878 (2017) 012031.

[43] K. Fukushima. Phys. Rev. C91 (2015) 044910 [1409.0698].

[44] V. Vovchenko, M. I. Gorenstein and H. Stoecker. Phys. Rev. Lett. 118 (2017) 182301 [1609.03975].

[45] V. Vovchenko, L. Jiang, M. I. Gorenstein and H. Stoecker. Phys. Rev. C98 (2018) 024910 [1711.07260]. 
[46] T. Dietrich, S. Bernuzzi, M. Ujevic and B. Brügmann. Phys. Rev. D91 (2015) 124041 [1504.01266].

[47] D. Radice, S. Bernuzzi, W. Del Pozzo, L. F. Roberts and C. D. Ott. Astrophys. J. 842 (2017) L10 [1612.06429].

[48] M. Hanauske, J. Steinheimer, A. Motornenko, V. Vovchenko, L. Bovard, E. R. Most et al. Particles 2 (2019) 44.

[49] M. Hanauske, L. Bovard, E. Most, J. Papenfort, J. Steinheimer, A. Motornenko et al. Universe 5 (2019) .

[50] R. C. Tolman. Phys. Rev. 55 (1939) 364.

[51] J. R. Oppenheimer and G. M. Volkoff. Phys. Rev. 55 (1939) 374.

[52] G. Baym, C. Pethick and P. Sutherland. Astrophys. J. 170 (1971) 299.

[53] L. Rezzolla, E. R. Most and L. R. Weih. Astrophys. J. 852 (2018) L25 [1711. 00314 ].

[54] R. S. Lynch, J. Boyles, S. M. Ransom, I. H. Stairs, D. R. Lorimer, M. A. McLaughlin et al. 763 (2013) 81 [1209.4296].

[55] S. Benic, D. Blaschke, D. E. Alvarez-Castillo, T. Fischer and S. Typel. Astron. Astrophys. 577 (2015) A40 [1411.2856].

[56] Y. Song, G. Baym, T. Hatsuda and T. Kojo. . 1905.01005.

[57] T. Hinderer, B. D. Lackey, R. N. Lang and J. S. Read. Phys. Rev. D81 (2010) 123016 [0 911.3535$].$

[58] M. Hanauske and L. Bovard. Journal of Astrophysics and Astronomy 39 (2018) 45. 